\title{
Constraints on New Physics in Electron $g-2$ from a Search for Invisible Decays of a Scalar, Pseudoscalar, Vector, and Axial Vector
}

Yu. M. Andreev, ${ }^{6}$ D. Banerjee, ${ }^{4}$ J. Bernhard, ${ }^{4}$ V. E. Burtsev, ${ }^{2}$ A. G. Chumakov, ${ }^{12,13}$ D. Cooke, ${ }^{5}$ P. Crivelli, ${ }^{15}$ E. Depero, ${ }^{15}$ A. V. Dermenev, ${ }^{6}$ S. V. Donskov, ${ }^{10}$ R. R. Dusaev, ${ }^{12}$ T. Enik, ${ }^{2}$ N. Charitonidis, ${ }^{4}$ A. Feshchenko, ${ }^{2}$ V. N. Frolov, ${ }^{2}$ A. Gardikiotis, ${ }^{9}$ S. G. Gerassimov, ${ }^{3,7}$ S. N. Gninenko®, ${ }^{6,}$ M. Hösgen, ${ }^{1}$ V. A. Kachanov, ${ }^{10}$ A. E. Karneyeu, ${ }^{6}$ G. Kekelidze, ${ }^{2}$ B. Ketzer, ${ }^{1}$ D. V. Kirpichnikov, ${ }^{6}$ M. M. Kirsanov, ${ }^{6}$ V. N. Kolosov, ${ }^{10}$ I. V. Konorov, ${ }^{3,7}$ S. G. Kovalenko, ${ }^{11}$

V. A. Kramarenko, ${ }^{2,8}$ L. V. Kravchuk, ${ }^{6}$ N. V. Krasnikov, ${ }^{2,6}$ S. V. Kuleshov, ${ }^{11,16}$ V. E. Lyubovitskij, ${ }^{12,13,14}$ V. Lysan, ${ }^{2}$ V. A. Matveev, ${ }^{2}$ Yu. V. Mikhailov, ${ }^{10}$ L. Molina Bueno, ${ }^{15}$ D. V. Peshekhonov, ${ }^{2}$ V. A. Polyakov, ${ }^{10}$ B. Radics, ${ }^{15}$ R. Rojas, ${ }^{14}$ A. Rubbia, ${ }^{15}$ V. D. Samoylenko, ${ }^{10}$ H. Sieber, ${ }^{15}$ D. Shchukin, ${ }^{7}$ V. O. Tikhomirov, ${ }^{7}$ I. Tlisova, ${ }^{6}$ A. N. Toropin, ${ }^{6}$

A. Yu. Trifonov, ${ }^{12,13}$ B. I. Vasilishin, ${ }^{12}$ P. V. Volkov, ${ }^{2,8}$ and V. Yu. Volkov ${ }^{8}$

(NA64 Collaboration)

\author{
${ }^{1}$ Universität Bonn, Helmholtz-Institut für Strahlen-und Kernphysik, 53115 Bonn, Germany \\ ${ }^{2} J o i n t$ Institute for Nuclear Research, 141980 Dubna, Russia \\ ${ }^{3}$ Technische Universität München, Physik Department, 85748 Garching, Germany \\ ${ }^{4}$ CERN, European Organization for Nuclear Research, CH-1211 Geneva 23, Switzerland \\ ${ }^{5}$ UCL Departement of Physics and Astronomy, University College London, Gower Street London WC1E 6BT, United Kingdom \\ ${ }_{6}^{6}$ Institute for Nuclear Research, 117312 Moscow, Russia \\ ${ }^{7}$ P.N. Lebedev Physical Institute, Moscow, Russia, 119991 Moscow, Russia \\ ${ }^{8}$ Skobeltsyn Institute of Nuclear Physics, Lomonosov Moscow State University, 119991 Moscow, Russia \\ ${ }^{9}$ Physics Department, University of Patras, 26504 Patras, Greece \\ ${ }^{10}$ State Scientific Center of the Russian Federation Institute for High Energy Physics of National Research Center "Kurchatov Institute" \\ (IHEP), 142281 Protvino, Russia \\ ${ }^{11}$ Departamento de Ciencias Físicas, Universidad Andres Bello, Sazié 2212, Piso 7, Santiago, Chile \\ ${ }^{12}$ Tomsk Polytechnic University, 634050 Tomsk, Russia \\ ${ }^{13}$ Tomsk State Pedagogical University, 634061 Tomsk, Russia \\ ${ }^{14}$ Universidad Técnica Federico Santa María, 2390123 Valparaíso, Chile \\ ${ }^{15}$ ETH Zürich, Institute for Particle Physics and Astrophysics, CH-8093 Zürich, Switzerland \\ ${ }^{16}$ SAPHIR Millennium Institute of ANID, 7591538 Santiago, Chile
}

(Received 6 February 2021; accepted 16 April 2021; published 25 May 2021)

\begin{abstract}
We performed a search for a new generic $X$ boson, which could be a scalar $(S)$, pseudoscalar $(P)$, vector $(V)$, or an axial vector $(A)$ particle produced in the $100 \mathrm{GeV}$ electron scattering off nuclei, $e^{-} Z \rightarrow e^{-} Z X$, followed by its invisible decay in the NA64 experiment at CERN. No evidence for such a process was found in the full NA64 dataset of $2.84 \times 10^{11}$ electrons on target. We place new bounds on the $S, P, V, A$ coupling strengths to electrons, and set constraints on their contributions to the electron anomalous magnetic moment $a_{e},\left|\Delta a_{X}\right| \lesssim 10^{-15}-10^{-13}$ for the $X$ mass region $1 \mathrm{MeV} \lesssim m_{X} \lesssim 1 \mathrm{GeV}$. These results are an order of magnitude more sensitive compared to the current accuracy on $a_{e}$ from the electron $g-2$ experiments and recent high-precision determination of the fine structure constant.
\end{abstract}

DOI: 10.1103/PhysRevLett.126.211802

Searching for new physics (NP) with mass below the electroweak scale $(\ll 100 \mathrm{GeV})$ at the high-intensity and

Published by the American Physical Society under the terms of the Creative Commons Attribution 4.0 International license. Further distribution of this work must maintain attribution to the author(s) and the published article's title, journal citation, and DOI. Funded by SCOAP ${ }^{3}$. high-precision frontiers has received significant attention in recent years [1-8]. Motivations for searches of feebly coupled particles in the low-mass range come from evidence for NP in the neutrino and dark matter sectors, and are well supported by theoretical arguments, see, e.g., Refs. [1,7-13]. Existing anomalies observed in particle experiments also contribute to the field. Well-known examples are the current muon $g-2$ anomaly-the $\simeq 3.6 \sigma$ discrepancy between the predicted and observed 
value of the muon anomalous magnetic moment [14], or the X17 anomaly - an excess of $e^{+} e^{-}$events in the ${ }^{8} \mathrm{Be}$ and ${ }^{4} \mathrm{He}$ nuclei transitions $[15,16]$, which might be explained by NP models at low-mass scale, see, e.g., Refs. $[17,18]$. These anomalies are being scrutinized in upcoming experiments at Fermilab and JPARC [19,20], and with NA64 at CERN [21-23], respectively.

Recently, a new puzzle indicating the possible presence of NP in the electron $g-2$ has emerged. The precise measurements performed at Laboratoire Kastler Brossel (LKB) with ${ }^{87} \mathrm{Rb}$ rubidium atoms report a new value for the fine-structure constant $\alpha^{-1}=137.035999206(11)$ with a relative accuracy of 81 parts per trillion [24]. This result improves the accuracy on $\alpha$ by 2.5 over the previous measurements performed at Berkeley with ${ }^{137} \mathrm{Cs}$ atoms [25] but, surprisingly, it reveals a $5.4 \sigma$ difference from this latest result. Using these measurements of the fine-structure constant, the standard model (SM) prediction of the anomalous magnetic moment of the electron, $a_{e}=$ $(g-2)_{e} / 2$ [26,27], is $1.6 \sigma$ lower and $-2.4 \sigma$ higher than the direct experimental measurement of $a_{e}^{\exp }$ [28]:

$$
\begin{gathered}
\Delta a_{e}=a_{e}^{\exp }-a_{e}^{\mathrm{LKB}}=(4.8 \pm 3.0) \times 10^{-13} \\
\Delta a_{e}=a_{e}^{\exp }-a_{e}^{B}=(-8.8 \pm 3.6) \times 10^{-13}
\end{gathered}
$$

for the LKB and Berkeley measurements, respectively. The errors on $\Delta a_{e}$ are dominated mostly by the uncertainty in $a_{e}^{\text {exp }}$. With new measurements and improved SM calculations, one hopes to clarify whether the deviations of Eqs. (1) and (2) are a result of yet unknown experimental errors, or it is a sign of new physics contributing $a_{e}$ [29]. This motivates recent significant efforts towards possible explanation of the deviation, in particular the discrepancy of Eq. (2), with a NP effect, see, e.g., Refs. [30-47].

In this Letter, we study the question of whether a new light $X$ boson could contribute $a_{e}$. We consider models with a generic $X$ in the sub-GeV mass range, which could be a scalar $(S)$, pseudoscalar $(P)$, vector $(V)$, or an axial vector $(A)$ particle feebly coupled to electrons. It is assumed that the $X$ decays predominantly invisibly, $\Gamma(X \rightarrow$ invisible $) / \Gamma_{\text {tot }} \simeq 1$, e.g., into dark sector particles, thus escaping stringent constraints placed today on the visible decay modes of the $X$ into SM particles from collider, fixed-target, and atomic experiments [48]. The most stringent limits on the invisible $X$ in the sub-GeV mass range are obtained, so far, for the $V$ case of dark photons coupled to electrons through the mixing with SM photons by the NA64 [49] and BABAR [50] experiments, leaving a large area of the parameter space for the generic $X$ still unexplored. Various aspects of such an invisible $X$ weakly coupled to leptons including possible phenomenological implications can be found in Refs. [1-8,45,51,52].

The $e-X$ interaction with coupling strength $g_{X}$ defined as $g_{X}=\varepsilon_{X} e$ (here $\varepsilon_{X}$ is a parameter and $e$ is the charge of the electron) is given for the $S, P, V, A$ cases by phenomenological Lagrangians:

$$
\begin{aligned}
\mathcal{L}_{S} & =g_{S} \bar{e} e S \\
\mathcal{L}_{P} & =i g_{P} \bar{e} \gamma_{5} e P \\
\mathcal{L}_{V} & =g_{V} \bar{e} \gamma_{\mu} e V_{\mu} \\
\mathcal{L}_{A} & =g_{A} \bar{e} \gamma_{\mu} \gamma_{5} e A_{\mu} .
\end{aligned}
$$

The corresponding one-loop contributions to $a_{e}$ induced by the diagrams shown in Fig. 1 are given by

$$
\begin{gathered}
\Delta a_{S}=\frac{g_{S}^{2}}{4 \pi^{2}}\left(\frac{m_{e}}{m_{X}}\right)^{2}\left[\ln \frac{m_{X}}{m_{e}}-\frac{7}{12}\right] \\
\Delta a_{P}=\frac{g_{P}^{2}}{4 \pi^{2}}\left(\frac{m_{e}}{m_{X}}\right)^{2}\left[-\ln \frac{m_{X}}{m_{e}}+\frac{11}{12}\right] \\
\Delta a_{V}=\frac{g_{V}^{2}}{4 \pi^{2}}\left(\frac{m_{e}}{m_{X}}\right)^{2} \frac{1}{3} \\
\Delta a_{A}=\frac{g_{A}^{2}}{4 \pi^{2}}\left(\frac{m_{e}}{m_{X}}\right)^{2}\left(-\frac{5}{3}\right)
\end{gathered}
$$

assuming that $m_{X} \gg m_{e}$. One can see that presumably a scalar and a vector can explain the positive deviation of Eq. (1), while only a pseudoscalar and an axial vector could explain the negative value of Eq. (2). The required couplings $g_{X}$ to explain deviations of Eqs. (1) and (2) are in the range $10^{-3} \lesssim\left|g_{X}\right| \lesssim 10^{-4}$ which is accessible to the NA64 search.

The method of the search, discussed in this work and proposed in Refs. [53,54], is based on the detection of the missing energy, carried away by the hard bremsstrahlung $X$ produced in the process $e^{-} Z \rightarrow e^{-} Z X ; X \rightarrow$ invisible of high-energy electrons scattering in an active beam dump. The NA64 experiment employed a $100 \mathrm{GeV}$ pure electron beam, using the $\mathrm{H} 4$ beam line of CERN's North Area. The beam was slowly extracted towards NA64 in 4.8 s spills, and had an intensity of up to $\simeq 10^{7}$ electrons per spill. A more detailed description of the NA64 detector can be found in Ref. [55] (see Fig. 1 therein). Below, its main features will be briefly mentioned. The $e^{-}$beam was defined by the scintillator $\left(\mathrm{Sc}_{1-4}\right)$ and veto $\left(V_{1,2}\right)$ counters.
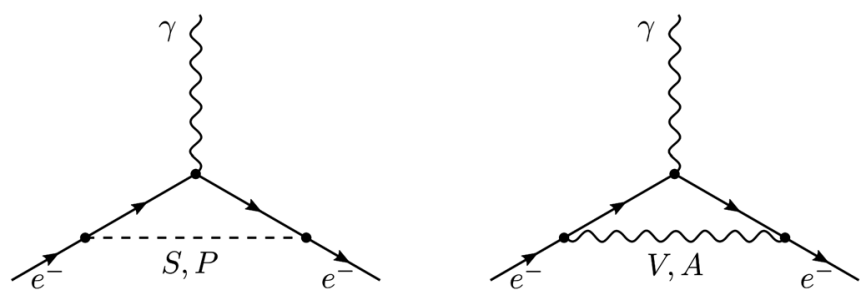

FIG. 1. One-loop contribution of the $S$ and $P$ (left panel) and the $V$ and $A$ (right panel) to $\Delta a_{e}$. 
A magnetic spectrometer consisting of two successive dipole magnets with the integral magnetic strength of $\simeq 7 \mathrm{~T} \cdot \mathrm{m}$ and a low-material-budget tracker consisting of a set of the Micromegas (MM), straw-tube (ST), and gaseous electron multiplier (GEM) chambers allowed us to measure the incoming $e^{-}$momenta with a precision $\delta p / p \simeq 1 \%$ [56]. Electrons were efficiently tagged from the synchrotron radiation (SR) emitted in the magnets, by using the SR detector (SRD) [57], which was an array of a Pb-Sc sandwich calorimeter of a fine segmentation. By using the SRD the intrinsic hadron contamination of the beam of the order of $\sim 1 \%$ was further suppressed to a negligible level. The downstream part of the detector was equipped with an electromagnetic (EM) calorimeter (ECAL), a matrix of $6 \times 6$ Shashlik-type modules assembled from $\mathrm{Pb}$ and $\mathrm{Sc}$ plates serving as an active beam-dump target for measurement of the electron energy $E_{\mathrm{ECAL}}$. Each ECAL module has $\simeq 40$ radiation lengths $\left(X_{0}\right)$ with the first $4 X_{0}$ serving as a preshower detector (PS). Further downstream the detector was equipped with a high-efficiency veto counter (VETO), and a hermetic hadronic calorimeter (HCAL) of $\simeq 30$ nuclear interaction lengths in total. The HCAL was used as an efficient veto against hadronic secondaries and also to detect muons produced in $e^{-}$interactions in the target.

The search described in this Letter uses the data samples of $n_{\mathrm{EOT}}=2.84 \times 10^{11}$ electrons on target (EOT), collected in the years 2016, 2017, and 2018 (runs I, II, and III, respectively) at beam intensities mostly in the range $\simeq(5-9) \times 10^{6} e^{-}$per spill with a hardware trigger accepting events with in-time hits in beam-defining counters $\mathrm{Sc}_{1-4}$ and energy in the PS exceeding the threshold $E_{\mathrm{PS}}^{\text {th }} \simeq 0.3 \mathrm{GeV}$, and energy in the ECAL below $E_{\mathrm{ECAL}}^{\text {th }} \simeq$ $80 \mathrm{GeV}[49,58,59]$. The missing energy events should have the incoming track momentum $P_{e} \simeq 100 \pm 3 \mathrm{GeV}$, the total ECAL energy $E_{\mathrm{ECAL}} \lesssim 80 \mathrm{GeV}$, and VETO and HCAL zero-energy deposition, defined as energy below the thresholds $E_{\mathrm{VETO}}^{\mathrm{th}} \simeq 1 \mathrm{MIP}$ (minimum ionizing particle) and $E_{\mathrm{HCAL}}^{\mathrm{th}} \simeq 1 \mathrm{GeV}$, respectively. Data from these three runs, were processed with selection criteria similar to the one used in Refs. [49,59] and finally analyzed as described below.

A detailed GEANT4 [60,61] based Monte Carlo (MC) simulation was used to study detector performance and signal acceptance, to simulate backgrounds and selection cuts. For calculations of the signal yield we used the fully GEANT4 compatible package DMG4 [62]. Using this package the production of $X$ in the process $e^{-} Z \rightarrow e^{-} Z X$; $X \rightarrow$ invisible has been simulated for each type of interaction listed in Eq. (3) with cross sections obtained from exact tree-level (ETL) calculations, see, e.g., Refs. [63-65]. The produced signal samples were processed by the same reconstruction program as the real data and passed through the same selection criteria. The total number $n_{X}$ of produced $X$ bosons per single electron on target was calculated as

$$
n_{X}\left(g_{X}, m_{X}, E_{0}\right)=\frac{\rho N_{A}}{A_{\mathrm{Pb}}} \sum_{i} n\left(E_{0}, E_{e}, s\right) \sigma_{X}\left(E_{e}\right) \Delta s_{i}
$$

where $\rho$ is density of the target, $N_{A}$ is the Avogadro's number, $A_{\mathrm{Pb}}$ is the $\mathrm{Pb}$ atomic mass, $n\left(E_{0}, E_{e}, s\right)$ is the number of $e^{ \pm}$in the EM shower at the depth $s$ (in radiation lengths) with energy $E_{e}$ within the target of total thickness $T$, and $\sigma\left(E_{e}\right)$ is the $X$ production cross section in the kinematically allowed region up to $E_{X} \simeq E_{e}$ by an electron with the energy $E_{e}$ in the reaction $e^{-} Z \rightarrow e^{-} Z X$; $X \rightarrow$ invisible. The latter depends in particular on the coupling and mass $g_{X}, m_{X}$, and the beam energy $E_{0}$. The $X$ energy distribution $d n_{X} / d E_{X}$ was calculated for each case by taking into account the corresponding differential cross section $\left\{\left[d \sigma\left(E_{e}, E_{X}\right)\right] /\left(d E_{X}\right)\right\}$, as described in Ref. [64]. An example of the simulated $X$ (or missing) energy spectrum in the target calculated by using the detailed simulation of EM shower development by GEANT4 is shown for the $P$ and $V$ cases in Fig. 2 for the mass $m_{X}=20 \mathrm{MeV}$. The expected number of $X$ events in our detector from the reaction $e^{-} Z \rightarrow e^{-} Z X$; $X \rightarrow$ invisible was determined for each $X$ interaction type also by comparison to the rare process of dimuon production, $e^{-} Z \rightarrow e^{-} Z \gamma, \gamma \rightarrow \mu^{+} \mu^{-}$, which has a well-known reaction rate. These events originate from a QED reaction in the ECAL, dominated by the hard bremsstrahlung photon conversion into dimuon pairs on a target nucleus and accompanied by small energy deposition in the HCAL, thus mimicking the $X \rightarrow$ invisible decay events below the two-MIP threshold. The reaction was also used as a benchmark process allowing us to verify the reliability of the MC simulation, correct the signal acceptance, and cross-check systematic uncertainties and the background estimate [49,59]. Good agreement was found between the observations and simulations. Using rare dimuon events as a cross-check for normalization to the signal modes cancels

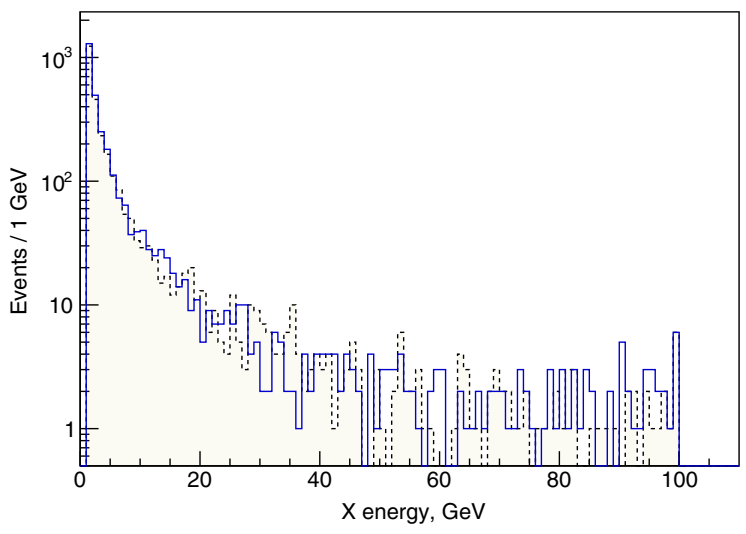

FIG. 2. The emission spectra of the $20 \mathrm{MeV} \mathrm{P}$ (solid line) and $V$ (dashed line) particles produced from the interactions of the $100 \mathrm{GeV}$ electron beam in the ECAL target obtained from the ETL calculations. The spectra are normalized to the same number of EOT. 
many systematic uncertainties by keeping selection cuts identical whenever possible.

In order to avoid biases in the determination of the selection criteria for signal events, a blind analysis similar to the one described in Ref. [49] was performed. The signal box $\left(E_{\mathrm{ECAL}}<50 \mathrm{GeV} ; E_{\mathrm{HCAL}}<1 \mathrm{GeV}\right)$ was defined based on the energy spectrum calculations for $X$ bosons emitted by $e^{ \pm}$from the EM shower generated by the primary $e^{-}$in the ECAL $[63,64]$ and the HCAL zero-energy threshold determined mostly by the noise of the read-out electronics. Finally, to maximize the acceptance for signal events and to minimize backgrounds we used the following selection criteria: (i) The incoming electron track momentum should be within $100 \pm 3 \mathrm{GeV}$. (ii) The SRD energy should be within the range of the SR energy emitted by $e^{-}$'s in the magnets and in time with the trigger. (iii) The shower shape in the ECAL should be consistent with that expected for the signal shower [63]. (iv) There should be only a single track activity in the tracker chambers upstream of the dump in order to reject interactions in the beam line material, and no activity in VETO.

The dominant background of $0.43 \pm 0.16$ events for $e^{-} Z \rightarrow e^{-} Z X ; X \rightarrow$ invisible arises from the interactions of the $e^{-}$beam in the downstream part of the detector resulting in hadron electroproduction in the beam line materials. In rare cases, these reactions are accompanied by the emission of large-angle (high $p_{T}$ ) hadronic secondaries faking the signal due to insufficient downstream detector coverage. The most of charged secondaries were rejected by requiring no additional tracks or hits in the downstream ST chambers, which have the largest transverse acceptance. We also requested no extra in-time hits upstream of the magnets and at most one extra in-time hit downstream of the magnets in the MM chambers. The remaining background of $0.38 \pm 0.14$ events from large-angle neutral hadronic secondaries was evaluated mainly from data by the extrapolation of events from the sideband $\left(E_{\mathrm{ECAL}}>\right.$ $50 \mathrm{GeV} ; E_{\mathrm{HCAL}}<1 \mathrm{GeV}$ ) into the signal region and assessing the systematic errors by varying the fit functions selected as described in Ref. [59]. The shape of the extrapolation functions was evaluated from the study of a larger data sample of events from hadronic $e^{-}$interactions in the dump, which was also cross-checked with simulations. Another background from punchthrough of leading (with energy $\gtrsim 0.5 E_{0}$ ) neutral hadrons $\left(n, K_{L}^{0}\right)$ produced in the $e^{-}$interactions in the target, was studied by using events from the region $\left(E_{\mathrm{ECAL}}<50 \mathrm{GeV} ; E_{\mathrm{HCAL}}>1 \mathrm{GeV}\right)$, which were pure neutral hadronic secondaries produced in the ECAL. Its level was estimated from the data by using the longitudinal segmentation of the HCAL and the punchthrough probability estimated conservatively and was found to be small. Several other background sources that may fake the signal, such as loss of dimuons due to statistical fluctuations of the signal or muon decays, and decays in flight of mistakenly SRD tagged beam $\pi, K$ were simulated with the full statistics of the data and were found to be negligible. After determining all the selection criteria and background levels, we unblinded the signal region and found 0 events consistent with $0.53 \pm 0.17$ events from the conservative background estimations [49] allowing us to obtain the $m_{X}$-dependent upper limits on the $e$-X coupling strengths.

The overall signal efficiency $\epsilon_{X}$, defined as the product of signal efficiencies accounting for the geometrical acceptance, the track, SRD, VETO, and HCAL reconstruction, and the DAQ dead time, was found to be slightly dependent on $m_{X}, E_{X}$ values [49]. The signal-event reconstruction efficiency $\epsilon_{\mathrm{ECAL}}$ was estimated as a function of energy deposited in the ECAL for different $X$ masses. Compared to the ordinary EM shower, the $\epsilon_{\mathrm{ECAL}}$ value for a shower from an $X$ event has to be corrected due to difference in the EM showers development at the early stage in the ECAL PS [63]. Depending on the energy threshold in the PS ( $E_{\mathrm{PS}}^{\mathrm{th}}$ ) used in the trigger this correction was $\lesssim(5 \pm 3) \%$ dominated by the errors due to the $E_{\mathrm{PS}}^{\text {th }}$ variation during the run. The VETO and HCAL efficiency defined by the leakage of the signal shower energy from the ECAL to these detectors, was studied for different $X$ masses with simulations that were validated with data measurements. The uncertainty in the efficiencies dominated mostly by pileup effects was estimated to be $\lesssim 4 \%$. The trigger efficiency was found to be $0.95 \pm 0.02$. The $X$ signal-event acceptance was estimated by taking into account the efficiency of the selection cuts for the signal shower shape in the ECAL [63]. The dominant uncertainty in the signal yield $\simeq 10 \%$ was conservatively assigned as the difference between the predicted and measured dimuon yield [59]. The total signal efficiency for beam intensity $\sim 5 \times 10^{6} e^{-}$per spill varied from $\sim 0.65$ to $\sim 0.5$ for the $X$ mass range from 1 to $200 \mathrm{MeV}$, decreasing for $m_{X} \lesssim 1 \mathrm{GeV}$ to $\sim 0.35$.

To set the limits we analyzed runs I-III simultaneously using the technique based on the RooStats package [66] allowing multibin limit setting [59]. For each of the $X=S$, $P, V, A$ cases, we tried to optimize the size of the signal box by comparing sensitivities defined as an average expected limit calculated using the profile likelihood method. The calculations were done by taking into account the background estimate, efficiencies, and their corrections with uncertainties used as nuisance parameters [67]. For this optimization, the most important inputs came from the background extrapolation into the signal region from the data samples of runs I-III with their errors estimated from the extrapolation procedure. The optimal signal box size was found to be weakly dependent on the $e-X$ type of interaction and $X$ mass varying with a few $\mathrm{GeV}$, and was finally set to $E_{\mathrm{ECAL}} \lesssim 50 \mathrm{GeV}$ for all four cases of Eq. (3) and the whole mass range. The total number of signal events was the sum of expected events from the all three runs in the signal box: 


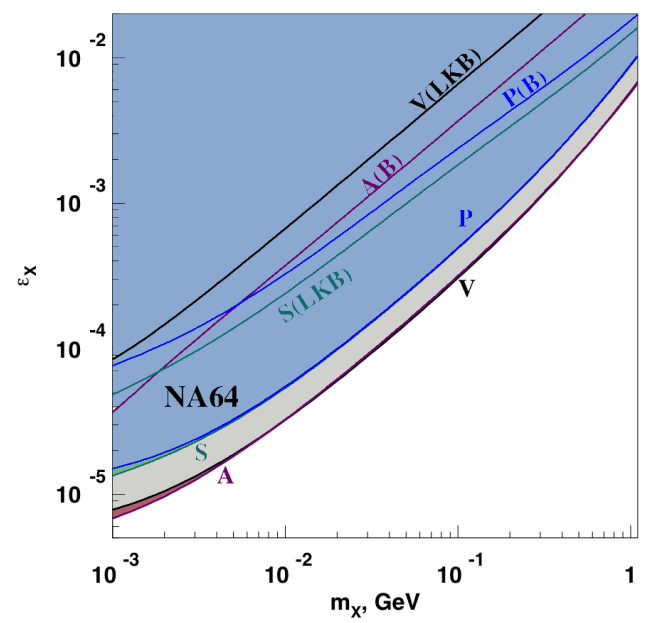

FIG. 3. The $90 \%$ C.L. upper limits on the coupling parameter $\varepsilon_{X}$ in the $\left(m_{X}, \varepsilon_{X}\right)$ plane obtained by NA64 and presented in comparison with the bounds derived from the results of the LKB [24] and Berkeley $(B)$ [25] experiments. The limits are shown by lines labeled with the $X$ type of the same color. The area above each line is excluded.

$$
N_{X}=\sum_{i=1}^{3} N_{X}^{i}=\sum_{i=1}^{3} n_{\mathrm{EOT}}^{i} \epsilon_{X}^{i} n_{X}^{i}\left(g_{X}, m_{X}, \Delta E_{e}\right),
$$

where $\epsilon_{X}^{i}$ and $n_{X}^{i}\left(\epsilon, m_{X}, \Delta E_{X}\right)$ are the signal efficiency and the signal yield per EOT in the energy range $\Delta E_{e}$, respectively. These values were calculated from simulations and processing of signal events through the reconstruction program with the same selection cuts and efficiency corrections as for the data sample from run $i$.

The combined $90 \%$ C.L. exclusion limits on the coupling parameter $\varepsilon_{X}$ as a function of the $X$ mass, calculated by using the modified frequentist approach $[49,68-70]$ are shown in Fig. 3. By using Eqs. (1) and (2) and (4)-(7), it is also possible to translate the measurements of Refs. [24,25] into constraints on the coupling $\varepsilon_{X}$ which are shown in Fig. 3 for comparison. The limits were calculated by taking into account the sign of the contributions $\Delta a_{X}$ in Eqs. (4)-(7) assuming that the $S$ and $V$ contribute to the deviation of Eq. (1), while only the $P$ and $A$ can resolve the discrepancy of Eq. (2). Our bounds are more stringent than those derived from the results of high-precision measurements of Refs. [24,25,28]. Using Eqs. (4)-(7) and obtained limits on the $X$ coupling strength we can derive constraints on the $X$ contribution $\Delta a_{X}$ to $a_{e}$. This results in stringent bounds in the range $\left|\Delta a_{X}\right| \lesssim 10^{-15}-10^{-13}$ for $S, P, V$, and $A$ with sub-GeV masses, which are shown in the $\left(m_{X} ;\left|\Delta a_{X}\right|\right)$ plane in Fig. 4 together with the experimental bands of the $\Delta a_{X}$ values defined by Eqs. (1) and (2). For the low mass region $m_{X} \lesssim 10 \mathrm{MeV}$ the limits were obtained by taking into account corrections from the exact calculations. These results are an order of magnitude more sensitive compared to the current accuracy on $a_{e}$ from the electron $g-2$

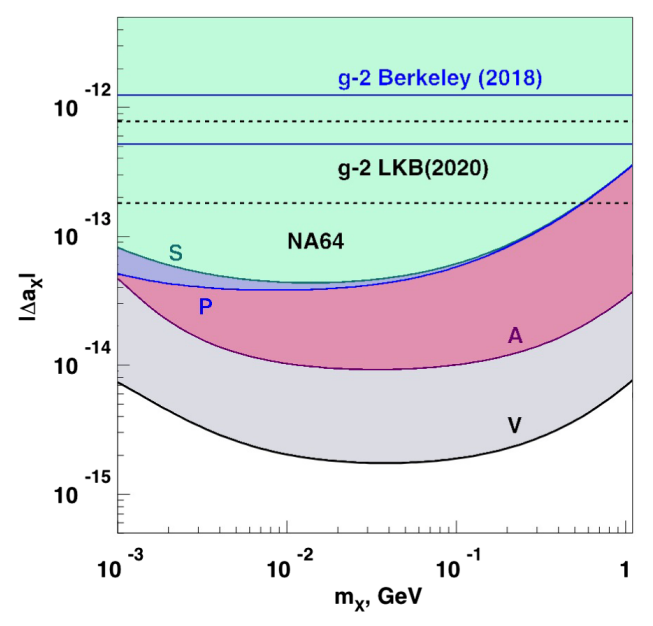

FIG. 4. Shown are the NA64 90\% C.L. exclusion region in the $\left(m_{X},\left|\Delta a_{X}\right|\right)$ plane for the $S, P, V$, and $A$ contributions to $a_{e}$ together with the bands of Eqs. (1) and (2), representing the results of the LKB [24] (black dashed lines) and Berkeley [25] (blue solid lines) experiments. The legend is the same as for Fig. 3. The area above each curve is excluded.

experiments and recent high-precision determination of the fine structure constant, thus demonstrating the strength of the NA64 approach of probing new physics in the electron $g-2$.

We gratefully acknowledge the support of the CERN management and staff and the technical staff of the participating institutions for their vital contributions. This work was supported by the Helmholtz-Institut für Strahlen-und Kern-physik (HISKP), University of Bonn, the Carl Zeiss Foundation 0653-2.8/581/2, and Verbundprojekt05A17VTA-CRESST-XENON (Germany), Joint Institute for Nuclear Research (JINR) (Dubna), the Ministry of Science and Higher Education (MSHE) and Russian Academy of Sciences (Russia), Swiss Federal Institute of Technology of Zurich and Swiss National Science Foundation Grants No. 197346, No. 186181, and No. 186158 (Switzerland), The National Fund for Scientific and Technological Development Grants No. 1191103 and No. 1190845, Millennium Institute for SubAtomic Physics at the High-energy Frontier Millennium Institute of the National Commission for Scientific and Technological Research (ANID) and ANID PIA/APOYO AFB180002 (Chile).

*Corresponding author. Sergei.Gninenko@cern.ch

[1] J. Jaeckel and A. Ringwald, Annu. Rev. Nucl. Part. Sci. 60, 405 (2010).

[2] R. Essig et al., arXiv:1311.0029.

[3] J. Alexander et al., arXiv:1608.08632.

[4] M. Battaglieri et al., arXiv:1707.04591.

[5] J. Beacham et al., J. Phys. G 47, 010501 (2020). 
[6] R. K. Ellis et al., arXiv:1910.11775.

[7] A. Berlin, N. Blinov, G. Krnjaic, P. Schuster, and N. Toro, Phys. Rev. D 99, 075001 (2019).

[8] G. Lanfranchi, M. Pospelov, and P. Schuster, arXiv:2011 .02157.

[9] C. Boehm and P. Fayet, Nucl. Phys. B683, 219 (2004).

[10] P. Fayet, Phys. Rev. D 75, 115017 (2007).

[11] M. Pospelov, A. Ritz, and M. B. Voloshin, Phys. Lett. B 662, 53 (2008).

[12] M. Pospelov, Phys. Rev. D 80, 095002 (2009).

[13] N. Arkani-Hamed, D. P. Finkbeiner, T. R. Slatyer, and N. Weiner, Phys. Rev. D 79, 015014 (2009).

[14] G. W. Bennett et al., Phys. Rev. D 73, 072003 (2006).

[15] A. J. Krasznahorkay et al., Phys. Rev. Lett. 116, 042501 (2016).

[16] A. J. Krasznahorkay et al., arXiv:1910.10459.

[17] S. N. Gninenko and N. V. Krasnikov, Phys. Lett. B 513, 119 (2001).

[18] J. L. Feng, B. Fornal, I. Galon, S. Gardner, J. Smolinsky, T. M. P. Tait, and P. Tanedo, Phys. Rev. Lett. 117, 071803 (2016).

[19] J. Grange et al., arXiv:1501.06858.

[20] T. Mibe, Chin. Phys. C 34, 745 (2010).

[21] D. Banerjee, V. E. Burtsev, A. G. Chumakov, D. Cooke, P. Crivelli et al. (NA64 Collaboration), Phys. Rev. Lett. 120, 231802 (2018).

[22] D. Banerjee et al. (NA64 Collaboration), Phys. Rev. D 101, 071101 (2020).

[23] E. Depero et al. (NA64 Collaboration), Eur. Phys. J. C 80, 1159 (2020).

[24] L. Morel, Zh. Yao, P. Cladé, and S. Guellati-Khélifa, Nature (London) 588, 61 (2020).

[25] R. H. Parker, C. Yu, W. Zhong, B. Estey, and H. Müller, Science 360, 191 (2018).

[26] T. Aoyama, M. Hayakawa, T. Kinoshita, and M. Nio, Phys. Rev. Lett. 109, 111807 (2012).

[27] T. Aoyama, T. Kinoshita, and M. Nio, Atoms 7, 28 (2019).

[28] D. Hanneke, S. Fogwell, and G. Gabrielse, Phys. Rev. Lett. 100, 120801 (2008).

[29] G. F. Giudice, P. Paradisi, and M. Passera, J. High Energy Phys. 11 (2012) 113.

[30] N. V. Krasnikov, Mod. Phys. Lett. A 35, 2050116 (2020).

[31] H. S. Lee, Phys. Rev. D 90, 091702(R) (2014).

[32] H. Davoudiasl and W. J. Marciano, Phys. Rev. D 98, 075011 (2018).

[33] W. J. Marciano, A. Masiero, P. Paradisi, and M. Passera, Phys. Rev. D 94, 115033 (2016).

[34] A. Crivellin, M. Hoferichter, and P. Schmidt-Wellenburg, Phys. Rev. D 98, 113002 (2018).

[35] E. J. Chun and T. Mondal, J. High Energy Phys. 11 (2020) 077.

[36] J. Liu, C. E. M. Wagner, and X.-P. Wang, J. High Energy Phys. 03 (2019) 008.

[37] M. Bauer, M. Neubert, S. Renner, M. Schnubel, and A. Thamm, Phys. Rev. Lett. 124, 211803 (2020).

[38] S. Gardner and X. Yan, Phys. Rev. D 102, 075016 (2020).

[39] L. Darme, F. Giacchino, E. Nardi, and M. Raggi, arXiv: 2012.07894 .
[40] L. D. Rose, S. Khalil, and S. Moretti, Phys. Lett. B 816, 136216 (2021).

[41] M. Endo and W. Yin, J. High Energy Phys. 08 (2019) 122.

[42] I. Dorsner, S. Fajfer, and S. Saad, Phys. Rev. D 102, 075007 (2020).

[43] K.-F. Chen, C.-W. Chiang, and K. Yagyu, J. High Energy Phys. 09 (2020) 119.

[44] C. Boehm, X. Chu, J.-L. Kuo, and J. Pradler, Phys. Rev. D 103, 075005 (2021).

[45] S. N. Gninenko, N. V. Krasnikov, and V. A. Matveev, Phys. Part. Nucl. 51, 829 (2020).

[46] S. Jana, V. P. K., W. Rodejohann, and S. Saad, Phys. Rev. D 102, 075003 (2020).

[47] S. Jana, V. P. K., and S. Saad, Phys. Rev. D 101, 115037 (2020).

[48] P. A. Zyla et al. (Particle Data Group), Prog. Theor. Exp. Phys. 2020, 083C01 (2020).

[49] D. Banerjee et al. (NA64 Collaboration), Phys. Rev. Lett. 123, 121801 (2019).

[50] J. P. Lees et al. (BABAR Collaboration), Phys. Rev. Lett. 119, 131804 (2017).

[51] S. N. Gninenko, D. V. Kirpichnikov, and N. V. Krasnikov, Phys. Rev. D 100, 035003 (2019).

[52] D. V. Kirpichnikov, V. E. Lyubovitskij, and A. S. Zhevlakov, Phys. Rev. D 102, 095024 (2020).

[53] S. N. Gninenko, Phys. Rev. D 89, 075008 (2014).

[54] S. Andreas et al., arXiv:1312.3309.

[55] D. Banerjee et al. (NA64 Collaboration), Phys. Rev. Lett. 125, 081801 (2020).

[56] D. Banerjee, P. Crivelli, and A. Rubbia, Adv. High Energy Phys. 2015, 105730 (2015).

[57] E. Depero et al., Nucl. Instrum. Methods Phys. Res., Sect. A 866, 196 (2017).

[58] D. Banerjee, V. Burtsev, D. Cooke, P. Crivelli, E. Depero et al. (NA64 Collaboration), Phys. Rev. Lett. 118, 011802 (2017).

[59] D. Banerjee, V. E. Burtsev, A. G. Chumakov, D. Cooke, P. Crivelli et al. (NA64 Collaboration), Phys. Rev. D 97, 072002 (2018).

[60] S. Agostinelli et al. (GEANT4 Collaboration), Nucl. Instrum. Methods Phys. Res., Sect. A 506, 250 (2003).

[61] J. Allison et al., IEEE Trans. Nucl. Sci. 53, 270 (2006).

[62] A. Celentano, M. Bondi, R. R. Dusaev, D. V. Kirpichnikov, M. M. Kirsanov, N. V. Krasnikov, L. Marsicano, and D. Shchukin, arXiv:2101.12192.

[63] S. N. Gninenko, N. V. Krasnikov, M. M. Kirsanov, and D. V. Kirpichnikov, Phys. Rev. D 94, 095025 (2016).

[64] S. N. Gninenko, D. V. Kirpichnikov, M. M. Kirsanov, and N. V. Krasnikov, Phys. Lett. B 782, 406 (2018).

[65] R. R. Dusaev, D. V. Kirpichnikov, and M. M. Kirsanov, Phys. Rev. D 102, 055018 (2020).

[66] I. Antcheva et al., Comput. Phys. Commun. 180, 2499 (2009).

[67] E. Gross, LHC statistics for pedestrians, CERN Report No. CERN-2008-001, 2008, p. 71.

[68] T. Junk, Nucl. Instrum. Methods Phys. Res., Sect. A 434, 435 (1999).

[69] G. Cowan, K. Cranmer, E. Gross, and O. Vitells, Eur. Phys. J. C 71, 1554 (2011).

[70] A. L. Read, J. Phys. G 28, 2693 (2002). 